\title{
Imaging of peripheral vascular disease
}

\author{
Mo Al-Qaisi' \\ David M Nott ${ }^{\prime}$ \\ David H King' \\ Sam Kaddoura ${ }^{2}$ \\ Mo Hamady ${ }^{3}$ \\ 'Charing Cross Hospital, London, UK; \\ ${ }^{2}$ Royal Brompton Hospital, London, UK; \\ ${ }^{3}$ St. Mary's Hospital, London, UK
}

\begin{abstract}
This illustrated review article gives an evidence-based update on the different modalities used for imaging peripheral vascular disease (duplex ultrasound, computed tomography angiography, magnetic resonance angiography, and digital subtraction angiography). After discussing the latest technological developments for each modality, their limitations are also highlighted. The evidence is presented for the various modalities' roles in the imaging of peripheral vascular disease, including problem-solving applications. The strengths and weaknesses of each modality are therefore critically appraised, including the salient technological, clinical, and financial aspects. This review allows the general and specialist practitioner to make an informed decision on how best to deploy imaging tests in peripheral vascular disease as part of an evidence-based approach. The article concludes with a rational imaging algorithm for the investigation of peripheral vascular disease.
\end{abstract}

Keywords: imaging, peripheral, vascular, duplex, angiography, arterial

\section{Introduction}

Peripheral vascular disease affects 27 million people in Europe and North America, with the majority asymptomatic or undiagnosed. The Joint American College of Cardiology and American Heart Association Guidelines ${ }^{1}$ provide extensive evidence-based recommendations for the diagnosis, investigation and management of peripheral vascular disease. The current role of imaging in peripheral vascular disease is summarized in Table 1.

The current state of imaging technology for peripheral vascular disease is discussed below under each modality: duplex ultrasound (DU); digital subtraction angiography (DSA); magnetic resonance angiography (MRA); and computed tomography angiography (CTA). As important as it is to understand the new solutions provided by advances in technology, it is also important to understand the residual limitations for each imaging modality.

\section{Duplex ultrasound}

Duplex systems have curvilinear $(2-5 \mathrm{MHz})$ and rectilinear (7-12 MHz) probes for aortoiliac and for femoropopliteal imaging, respectively. Real-time B-mode imaging in combination with color Doppler and pulsed-wave Doppler waveform sampling provide both anatomical and hemodynamic (functional) information. DU is widely available, inexpensive, mobile, noninvasive, nonionizing, and does not require nephrotoxic contrast.

Duplex ultrasound has been tested (against DSA as gold-standard) in patients with peripheral vascular disease and found to have good agreement for detecting significant stenoses and occlusions (sensitivity $70 \%$, specificity $98 \%$, positive predictive value 0.82 , negative predictive value 0.95 , and $\kappa$-value of 0.72 ). When the arterial tree was further sub-classified into pelvic, thigh, and calf regions, DU was found to have a high negative predictive value ( 0.99$)$ for the pelvic region - potentially ideal as a secondary sign for confirming aortic disease. ${ }^{2}$ DU also has an overall accuracy of $88 \%$ in the surveillance of lower limb venous bypass grafts. ${ }^{3}$ 
Table I Summary of Joint Guidelines' for imaging of peripheral vascular disease

\begin{tabular}{lll}
\hline Modality & Level I & Level 2 \\
\hline DSA & - Information on arterial inflow and outflow prior to & - Noninvasive imaging techniques may be used to plan digital \\
& decision on bypass or angioplasty & subtraction angiography (access site, lesion location) \\
MRA & - Anatomic location/degree of stenosis & - Selection of patients for bypass and sites of target vessels \\
& - Should be performed with gadolinium enhancement & - Surveillance of patients postbypass or postangioplasty \\
& - Selection of patients for angioplasty & \\
CTA & & - Anatomic location of significant stenosis ( $>50 \%)$ \\
Duplex ultrasound & - Anatomic location/degree of stenosis & - Substitute for MRA if MR contraindicated \\
& - Routine surveillance after bypass with venous & - Selection of patients for angioplasty \\
& conduit & - Selection of patients for bypass and sites of target vessels \\
& & - Can be considered for routine surveillance after \\
& & femoralpopliteal bypass with synthetic conduits \\
& & - Not well established for long-term patency \\
\end{tabular}

Notes: Level I, evidence-based peer agreement that a given investigation is indicated; Level 2, conflicting evidence or controversial peer opinion about the use of a given investigation.

Abbreviations: CTA, computed tomography angiography; DSA, digital subtraction angiography; MRA, magnetic resonance angiography.

\section{Limitations of duplex ultrasound}

Ultrasound has several operator-dependent and operatorindependent limitations. Ultrasound is a focal imaging tool, and cannot define co-existent extrinsic pathology completely or provide global imaging of the cardiovascular system. Ultrasound cannot provide reliable imaging if there are poor acoustic windows (eg, bowel gas attenuation, diffuse vascular calcification, or metallic stents) or poor intrinsic echogenicity of the tissues. Duplex scanning is time-consuming and inconvenient post-operatively - when the region of interest is obscured by dressings - and removing these can involve infection risk. A lack of vascular ultrasound expertise limits the use of DU for referral during working hours.

The sensitivity of DU was found to vary widely (from $46 \%$ to $88 \%$ ) for detecting significant arterial stenoses, depending on which arterial segment was being examined. ${ }^{4} \mathrm{DU}$ failed to visualize up to $10 \%$ of aortoiliac, $2 \%$ of femoropopliteal, and $13 \%$ of tibioperoneal arterial segments. The sensitivity of DU is as low as $72 \%$ in the pelvic region for detecting significant obstructions. ${ }^{5}$ In addition, some authors claim DU is not reliable for clinical decisions based on visualization of run-off vessels, ${ }^{2}$ or for evaluating residual stenosis after peripheral angioplasty. ${ }^{6}$ Figure 1 shows an example of DU imaging.

\section{Digital subtraction angiography}

Digital subtraction angiography provides the gold-standard for imaging in peripheral vascular disease, especially prior to surgical intervention. ${ }^{7}$ Digital cine-flouroscopy allows real-time guidance of interventional procedures which are first-line management for critical ischemia, especially in patients who are not fit for a general anesthetic.
Modern angiography suites consist of ultra-rapid digital cine-fluoroscopy systems, mounted on C-arms, with digital display facilities and complex post-processing software. The most critical step is the computerized subtraction of the precontrast (mask) image from the post-contrast image, producing a resultant image of contrast-filled vessels only. Digital subtraction techniques reduce the dose of contrast required. Good spatial and temporal resolution, combined with a large field of view, allows bolus chasing from the aorta to the feet. The peripheral arterial tree can be seen and mapped quickly with overlapping runs. Large numbers of collaterals can be anatomically visualized quickly and in one session (compared to the challenge of mapping collaterals accurately with DU).

More recently, rotational computed angiography (Dyna-CT) has been introduced. This involves a modified angiography image intensifier, with a rotating $\mathrm{C}$-arm, so that computed tomography-like slices can be acquired in the interventional suite.

\section{Limitations of digital subtraction angiography}

Disadvantages of DSA include the necessity of arterial access. Arterial puncture is a relative burden (if the same diagnosis could be made without it), especially if the patient does not benefit from an endovascular intervention at the same time. DSA involves ionizing radiation (for both patients and staff) and nephrotoxic contrast. The mask projections are easily distorted by patient movement, although modern software systems compensate for some motion artefacts. Slow flow can be missed depending on technical parameters (contrast injection rate, projection, or frame rate). 


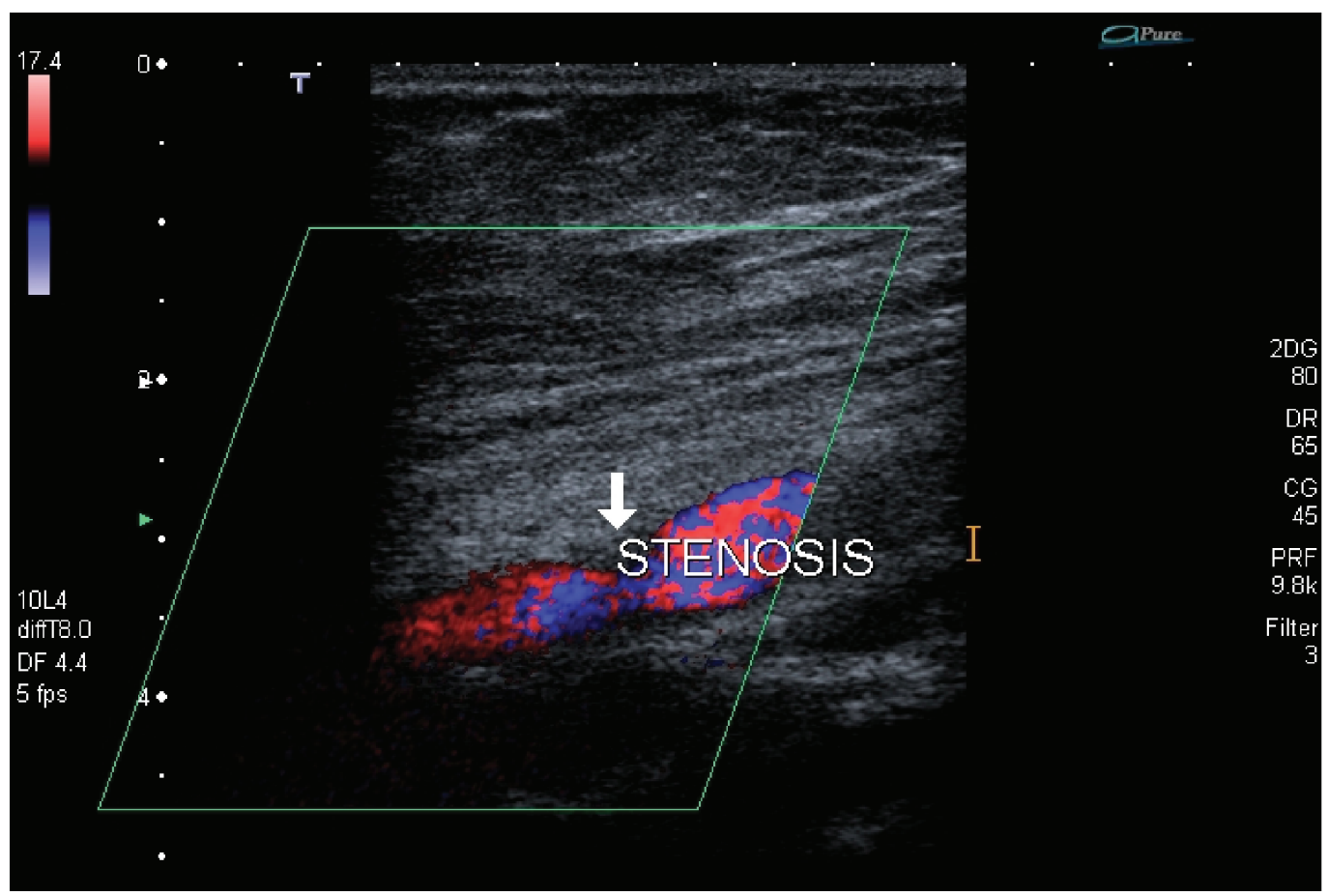

Figure I Proximal superficial femoral artery stenosis. Color flow ultrasound imaging of a diseased superficial femoral artery. Conventionally, red flow is towards the transducer and blue flow is away from the transducer. In this case, because of the position of the transducer, red is also flow towards the periphery, and blue is reverse flow. A short region of narrowing (marked with white arrow) generates a high velocity jet at the narrowest point and immediately downstream (towards right of arrow). The poststenotic flow is characterized by irregular, scintillating color changes from red through blue, the effect being primarily due to to aliasing, an artefact arising from blood velocities which exceed the maximum velocity scale setting, but also from turbulence. Similarly, near both walls of the artery, disturbed blood flow manifests as a mixture of forward (red) and reverse (blue) flow. These color changes are more regular and continue for a short distance downstream as the jet slows, becoming unstable in the process.

Finally, DSA is a form of luminal imaging, with information about extrinsic pathology deducible only indirectly (or not at all). Eccentric or en face plaque can be missed, ${ }^{7}$ and there is some inter-observer variation in grading stenoses. The latter can be reduced by using the quantitative tools in modern vascular software packages. Figure 2 shows an example of DSA images.

\section{Magnetic resonance angiography}

Magnetic resonance angiography consists of moving-table bolus-chase protocols using gadolinium-enhancement in combination with a phased-array leg angiography coil. Most tertiary vascular centers now have access to a dedicated magnetic resonance service, with fewer peripheral centers able to offer diagnostic expertise in this field. A three-step protocol with contrast enhancement is reputed to have higher diagnostic accuracy than two-dimensional (2D) time of flight MRA. ${ }^{9,10}$

Magnetic resonance angiography is ideal for problemsolving imaging of the below-knee run-off, which is not easily assessed by DU or DSA (problem-solving refers to the imaging in cases where the first-line modality cannot provide the diagnostic answers). Parallel imaging and innovative $\kappa$-space re-ordering have allowed sub-millimeter isotropic voxel acquisition in the distal run-off stations. "Hybrid" MRA is the combination of a time resolved MRA of the calf and foot followed by a bolus-chase multistation MRA. Time resolved imaging can be 2D or 3D. Hybrid MRA can detect run-off arteries not seen by DSA, which is of supreme importance in planning limb salvage surgery. ${ }^{11}$

Magnetic resonance angiography can provide a global assessment of the vascular system with relative ease. Additional unsuspected lesions (stenoses, aneurysms, or dissections) have been found in up to $20 \%$ of peripheral vascular patients. Vessel-wall specific sequences (eg, fatsaturated T1 gradient-echo, contrast-enhanced inversion recovery gradient echo) can be used to delineate some of these additional lesions with little increase in scan time. In addition, dark-blood HASTE (half-fourier acquisition single-shot turbo spin-echo) imaging is used for the aortic wall, however this technique has not been widely used or accepted for peripheral arterial imaging. ${ }^{12}$ Unlike DU, MRA allows simultaneous angiographic as well as anatomical volume acquisition of the whole body in seconds whilst maintaining high spatial resolution. This allows both luminal and extra-luminal pathology to be shown simultaneously, although magnetic resonance imaging is poorer for bony anatomy than computed tomography. 
A)

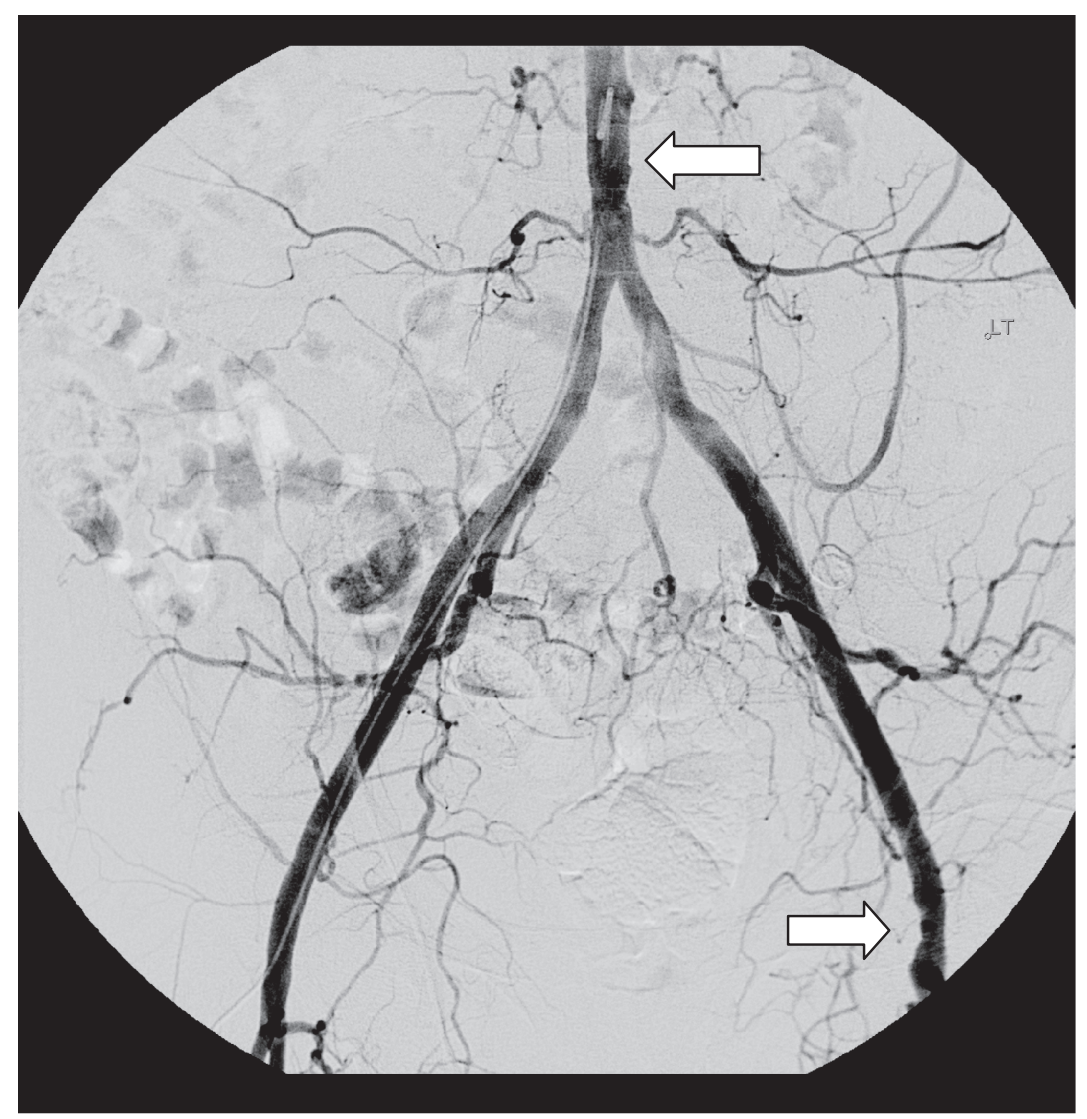

B)

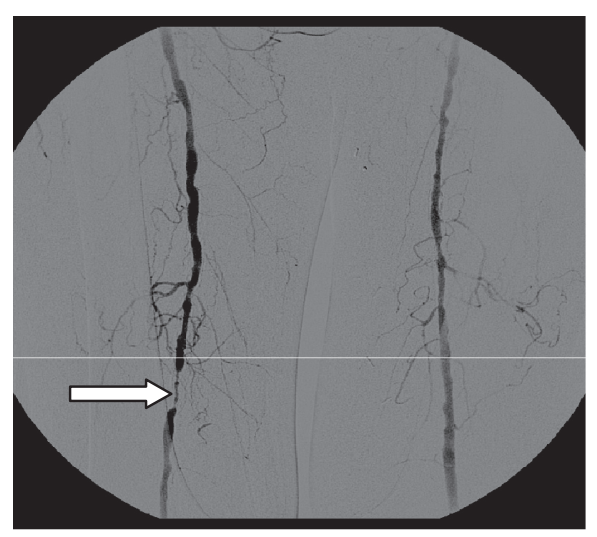

Figure 2 Digital subtraction angiograms. A) A catheter has been passed from the right common femoral artery and into the abdominal aorta during a digital subtraction angiogram. Contrast injection through the catheter reveals minor atherosclerotic wall abnormalities in the infrarenal aorta and the left common femoral artery (see arrows). B) Lower limb images reveal diffuse atherosclerotic disease of the superficial femoral and popliteal arteries of both sides, with a focal area of significant stenosis (arrow) in the right popliteal artery.

It has been claimed that MRA has a higher sensitivity than DU in detecting significant stenosis in any arterial segment (pelvic, femoral, or calf). ${ }^{2}$ In a prospective trial with 295 patients, using catheter angiography as a gold-standard, it was found that MRA was more sensitive ( $84 \%$ vs $76 \%$ ) and more specific (97\% vs $93 \%$ ) than DU. In particular, MRA was found to be superior in the aorto-iliac segment, where bowel gas confounds ultrasound techniques. ${ }^{13}$ 
Contrast enhancement not only improves the signal-to-noise ratio but also reduces imaging time. Contrast-enhanced MRA is similar or superior in accuracy to DU for the assessment of patency in lower limb bypass grafts. ${ }^{14}$ If it were possible to use MRA instead of DU in every case - which it is not, as many patients have contra-indications or sub-optimal magnetic imaging - it has been claimed that this would reduce the requirement for additional vascular imaging procedures by up to $42 \%{ }^{15}$

Invasive (intra-arterial contrast-enhanced) MRA has been studied by several groups. Intra-arterial enhancement required only $5 \%$ of the conventional gadolinium dose. ${ }^{16}$ When comparing invasive MRA with conventional DSA, there was a sensitivity of $93 \%$ and specificity of $86 \%$ for significant stenoses. The overall accuracy of the invasive method was lowest for the infrapopliteal segment (86\%) and highest (93\%) for the femoropopliteal region. ${ }^{17}$

\section{Limitations of magnetic resonance angiography}

Like DSA, contrast enhanced magnetic resonance imaging is sensitive to patient motion artefacts. If there is patient motion between the mask acquisition and postcontrast acquisition, there will be image degradation following subtraction. However, evaluation of the nonsubtracted source images is always part of the image interpretation and unlike DSA, purely unenhanced MRA is also possible.

The relative high cost and limited availability of services (especially after working hours) restricts the clinical utility of MRA. MRA involves strong magnetic fields, creating many contraindications (eg, pacemakers).

Long scan times (in a narrow tunnel) are problematic for claustrophobic patients, and sedation is sometimes necessary. Magnetic resonance cannot image calcification or steel stents; although the former can be an advantage in some circumstances, the latter causes susceptibility artefacts easily recognizable as signal voids in the image. Metallic structures within the vessel lumen or adjacent to the vessel may cause magnetic susceptibility artefacts that obscure the vessel lumen leading to a false diagnosis of a stenosis or obstruction.

The most serious limitation of MRA is venous contamination. The application of a compression cuff to the thigh (inflated to pressures of up to $50 \mathrm{mmHg}$ ) can reduce venous contamination. ${ }^{18}$ Some groups have reported a reduction in venous contamination with "hybrid" protocols using time resolved imaging. In addition, several 3D time-resolved protocols, including "TRICKS" (time-resolved imaging of contrast kinetics), "TREAT" (time-resolved echo-shared angiographic technique), and "TWIST" (time-resolved angiography with interleaved stochastic trajectories) have been shown to minimize venous contamination in the calf and pedal vessels. ${ }^{19-21}$ With all these techniques, temporal resolution is improved at the cost of reduced spatial resolution, and research is now focusing on how to improve on this tradeoff. ${ }^{21}$ Despite improved protocols, technically poor magnetic resonance studies occur in $13.7 \%$ of all patients. ${ }^{22} \mathrm{Up}$ to $33 \%$ of clinicians will still request DSA after MRA in order to make a therapeutic decision. ${ }^{23}$ In addition, MRA overestimates stenoses in up to $7 \%$ of cases when compared to DSA as the gold-standard.$^{24}$ However DSA itself may underestimate true lesion grade if used as gold-standard as has previously been implied from direct intra-arterial pressure measurements. ${ }^{5}$ High-field (3.0 Tesla) imaging is also being investigated for clinical applications, and improvements in the technology may well resolve some of these current limitations.

The use of gadolinium-based contrast agents has recently been associated with nephrogenic systemic fibrosis in patients with renal impairment (all cases have been in patients with a glomerular filtration rate of less than $60 \mathrm{ml} / \mathrm{min}$ ). The European Society of Urogenital Radiology (see http://www. esur.org) and the American College of Radiology Blue Ribbon Panel on Magnetic Resonance Safety (see http://www.acr. org) have both issued detailed guidance on this issue.

The concerns arising from gadolinium-based contrast agents have stimulated renewed interest in unenhanced techniques. Traditional unenhanced MRA techniques include phase contrast, time of flight, and black-blood vessel wall (double inversion recovery) imaging. All of these techniques have inferior accuracy to the newer 3D-contrast-enhanced techniques, and are therefore no longer used for clinical decision making. More recently, a new approach to unenhanced MRA has evolved using "fresh blood" imaging. With this method, electrocardiogram (ECG)-gated 3D imaging allows subtraction of diastolic images from systolic images, eliminating venous and tissue signal, without the need for contrast. It remains to be seen if "fresh blood" imaging is robust enough for clinical decision making, and what, if any, is the clinical utility of this approach. ${ }^{21}$ Figure 3 shows an example of MRA imaging.

\section{Multidetector computed tomography angiography}

Computed tomography angiography is widely available, including after working hours. It can be used for trauma, vascular emergencies, aneurysms, and for imaging bypass grafts. It is relatively less costly, and does not require on-site sub-specialist expertise or arterial puncture. It is suitable for 


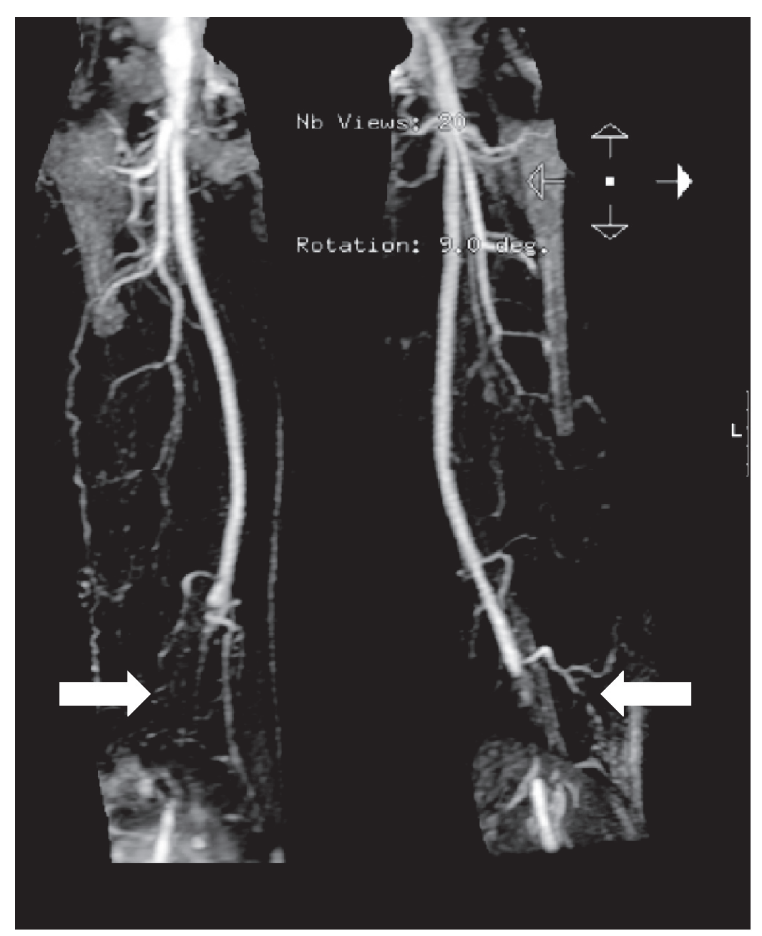

Figure 3 Contrast-enhanced magnetic resonance angiography (MRA) of lower limbs. Gadolinium-enhanced MRA shows bilateral femoro-popliteal occlusions (arrows). Using a subtraction technique, arterial phase images show the occlusions clearly.

preoperative planning, as well as the postoperative patient when bandages and sutures (not to mention edema) might make ultrasound difficult or undesirable (for example because of infection risk). Unlike DU, computed tomography allows simultaneous angiographic as well as anatomical volume acquisition in seconds whilst maintaining high spatial resolution. This allows both luminal and extraluminal pathology to be shown simultaneously.

Multidetector computed tomography scanners are rapid, and imaging can be done whilst contrast is in the first-pass phase of the arterial tree, so the venous contamination seen with MRA does not occur. ${ }^{25}$ Cardiac gating improves the accuracy of thoracic aorta imaging by minimizing motion artefacts.

Computed tomography is ideal for preoperative planning of aortoiliac stent-graft placement, as well as poststent surveillance. Up to $28 \%$ of endoleaks are missed by DU (and the proportion of missed endoleaks goes up to $42 \%$ if considering type II only). ${ }^{26} \mathrm{CTA}$ can provide rapid and accurate imaging of en face plaque in the aortoiliac segments. ${ }^{27}$ Similarly, computed tomography allows accurate assessment of mural thrombus in the aortoiliac region, providing the exact diameter of aneurysms, and exquisite images of the aortic wall and regional structures. ${ }^{25}$

Three separate systematic reviews of the diagnostic accuracy of CTA for peripheral arterial disease found a pooled sensitivity and specificity of $92 \%$ and $91 \%$ in one analysis, and $92 \%$ and $93 \%$ in the second, whereas the third reported 95\% and $96 \%$ respectively (all three systematic reviews used DSA as gold-standard). ${ }^{28-30}$ It should be noted that the first two of these systematic reviews included only two papers (out of 10 and 12, respectively) with 16-detector technology; all the other results were from 4-detector technology. ${ }^{28,29}$ In the third systematic review, there were seven papers (out of 20) with 16-detector technology, and two papers with 64-detector technology. ${ }^{30}$ The first published results with 16-detector technology claim overall accuracy, sensitivity, and specificity of over $96 \%$, without any decrease in performance down to the crural branches. ${ }^{31}$

More recent studies with 16-detector technology suggest sensitivity and specificity of appropriately $98 \%$ for peripheral vascular disease. ${ }^{32,33}$ The two studies presenting data for 64-detector technology (reported in the third meta-analysis) claimed a sensitivity and specificity for CTA of around 99\% for both indicators (in the first study) versus $98 \%$ and $96 \%$ respectively (in the second study). ${ }^{34,35}$

The current consensus position of the Joint American College of Cardiology and American Heart Association Guidelines $^{1}$ is that using CTA is controversial. This position is based on the technological lag and poor sensitivity of 4-detector scanners, and to some extent, 16-detector scanners. As the accuracy of CTA with 64-detector, 128-detector, 256-detector, and 320-detector (or more) machines emerges, the accepted consensus position on its use in the investigation of peripheral vascular disease is likely to change. It has been pointed out that the accuracy of CTA may be even higher than reported because a multi-planar 3-dimentional technology is being compared with a bi-planar (at best) or mono-planar methodology of image analysis for the gold-standard (DSA). In this case, CTA may be sensitive to disease missed by the gold-standard. ${ }^{30}$

From 64-detector onwards, computed tomography machines have isotropic acquisition and multiplanar $3 \mathrm{D}$ reconstruction capabilities, with much improved scan optimization protocols. Given the speed of imaging and the number of slices acquired with multidetector technology, it is not possible to handle the images by reviewing axial slices only. Fortunately, continuous advances in imaging informatics has allowed postprocessing capability to keep up with the filing and transfer of the vast amount of data produced by multidetector computed tomography. Various postprocessing algorithms (including surface shaded display, volume rendering, multiplanar reconstruction, vessel density ratio, and maximum intensity projection) facilitate the radiological analysis (albeit with 
limitations, see below) and reduce the work-flow pressure on radiographic technicians in busy imaging departments. ${ }^{25}$

The traditional route of access for contrast enhancement in computed tomography is venous. A study of intra-arterial contrast injection found an $88 \%$ reduction in contrast dose, ${ }^{36}$ making the invasive approach a potentially attractive option for patients with renal impairment, especially if MRA is contraindicated, unavailable, or technically unsuccessful.

\section{Limitations of computed tomography angiography}

Computed tomography imparts a nonnegligible dose of ionizing radiation dose to patients, but with the latest technology and optimal technical factors, this radiation dose can be comparable with DSA. A typical mean effective dose for CTA study of the periphery (including lower limbs) has been reported as $12-14 \mathrm{mSv}$ per study depending on technical protocol), ${ }^{37,38}$ versus $11 \mathrm{mSv}$ for DSA. ${ }^{31}$ Computed tomography generates comprehensive anatomic volume acquisition, and this requires not only post-processing time but also formal reporting of all the voluminous imaging. Being an iodine-based contrast technique, there is a recognized associated nephrotoxicity and adverse reaction rate; and being diagnostic only, the requirement for nephrotoxic contrast needs to be considered carefully in patients who have poor renal function. In these cases, it might be necessary to preserve the little remaining nephrotoxic reserve and proceed directly to DSA with the chance of simultaneous angioplasty and definitive management.

Extensive vessel wall calcification makes assessment of luminal stenosis very difficult with current computed tomography techniques. Patients with heavy calcification have better diagnostic outcomes with MRA or DSA. Calcification confounds the software-driven rendering and automation used in computed tomography, and images become particularly poor for the distal run-off in the presence of calcific disease, which is a limitation in common with DU.

Interestingly, the third meta-analysis (mentioned previously) reported that CTA overstaged $8 \%$ and understaged $15 \%$ of diseased arterial segments. ${ }^{30}$ Overstaging can occur because of the "blooming" effect of the calcium in calcified plaques, so that a patent vessel with calcification is mistaken for an occluded vessel on the CT images. ${ }^{39}$ Understaging occurs when DSA misdiagnoses patent vessels as occluded because of motion artefacts, different rates of crural vessel opacification, or inadequate opacification distal to an occlusion. ${ }^{40}$ For this reason, CTA should be avoided in the elderly ( $>84$ years), patients with renal failure, diabetes, or heart failure. ${ }^{41}$ Similarly, in the setting of trauma, metal fragments can cause artefactual "pseudolesions", whilst vessel spasm can be difficult to distinguish from occlusion. ${ }^{42}$ Metallic aneurysm clips and stents attenuate the X-ray beam, allowing too few photons to reach the X-ray detectors, and corrupting the system's ability to map the projection. Those few photons that do reach the detectors are the ones with higher energies, therefore resulting in "beam hardening". Traditional filtered back-projection algorithms used to reconstruct spiral computed tomography images are degraded by streaking and "star artefacts" in the plane of a metallic implant. Recent research is focusing on how to limit metallic artefacts using novel reconstruction algorithms. ${ }^{43}$

Other limitations of computed tomography relate to the postprocessing algorithms used to facilitate analysis of the images. Maximum intensity projections can mask co-existent subtle trauma, and both maximum intensity projections and vessel density ratios exaggerate the extent of stenosis in the presence of calcified plaque. In addition, multiplanar reconstruction can be poor for configuring tortuous or off-axis arteries. ${ }^{25}$ Figure 4 shows an example of CTA imaging.

\section{Comparative resource implications}

Studies of health care economics, quality, and outcomes are notoriously fraught with limitations. Results can vary widely, and incorporating cost adjustments for the quality of a study is far from an exact science. One group in the Netherlands recently attempted to quantify the comparative average cost and outcome based on choice of the first noninvasive imaging test in patients with peripheral vascular disease. The average MRA was found to be much more expensive (at 472 Euros/study) than both CTA (163 Euros/study) and DU (104 Euros/study). Diagnostic-only digital subtraction angiograms were estimated to cost 1207 Euros/study. Being investigated with CTA as the first-line study appeared to be much less costly overall (including the costs of additional tests required) than having MRA or DU as first-line. However, despite these different costs, the group could not find any difference between the functional or quality outcomes of patients investigated by any of the techniques as first-line. ${ }^{44}$

\section{Clinical algorithm for investigating peripheral vascular disease}

A complementary imaging strategy would deploy the different imaging modalities to provide the most efficient diagnostic work-up of a patient, providing complete diagnostic information to clinical decision-makers. Each imaging modality provides a different perspective of the same clinical 


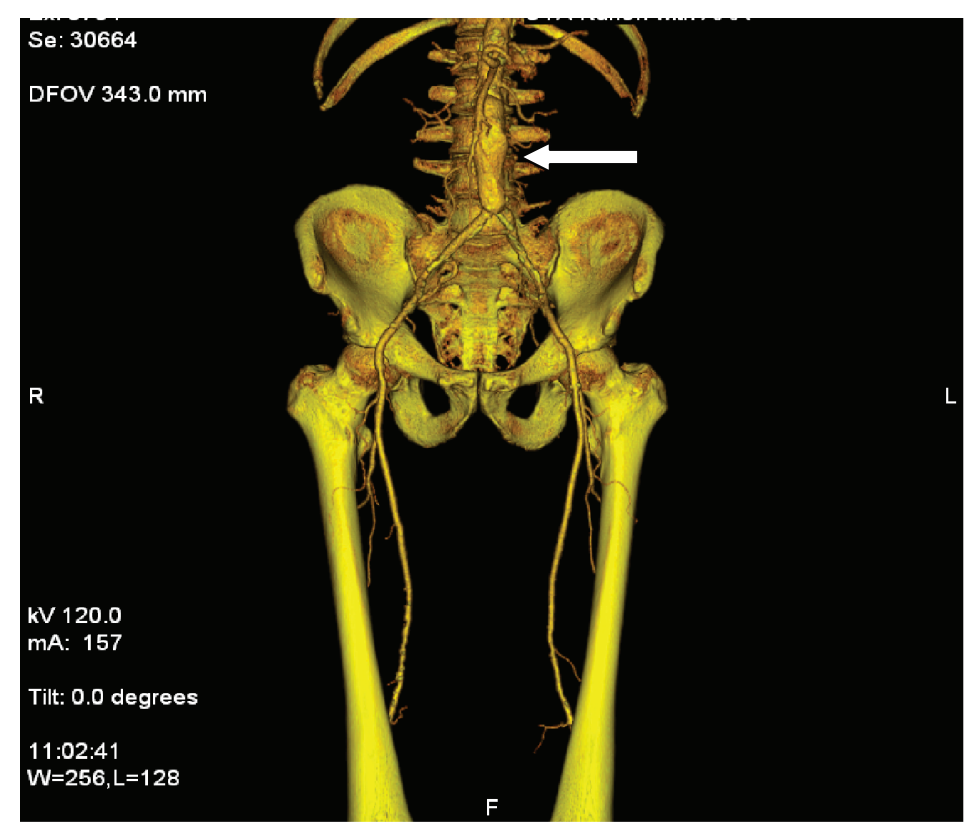

Figure 4 Volume rendered computed tomography angiography (CTA) of lower limbs. Software-driven volume-rendering shows dramatic three-dimensional (3D) reconstruction possible with new CT machines. The software packages for processing CT data can be selected to display only the arterial tree and skeletal structures. The 3D images can be rotated and are helpful in the rapid diagnostic evaluation of patients with emergency or trauma presentations. The volume-rendered image reveals an abdominal aortic aneurysm (arrow) in the infrarenal aorta and calcified plaques in the common and external iliac arteries. There is additional atherosclerotic disease in the distal segment of the right superficial femoral artery.

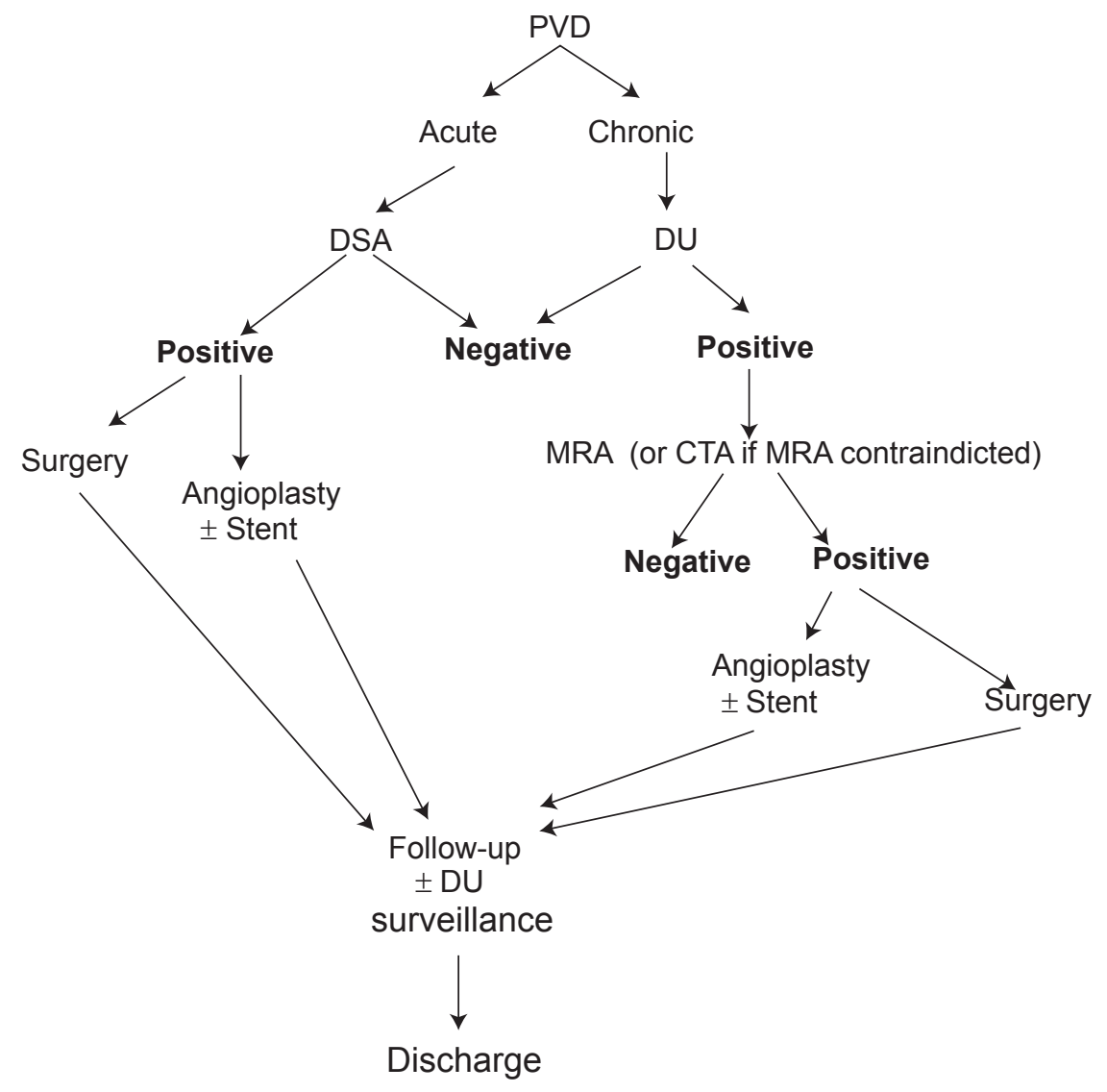

Figure 5 Clinical algorithm for imaging of peripheral vascular disease.

Abbreviations: PVD, peripheral vascular disease; DSA, digital subtraction angiography; DU, duplex ultrasound; MRA, magnetic resonance angiography; CTA, computed tomography angiography. 
Table 2 Role of different imaging techniques in the investigation of PVD

\begin{tabular}{lll}
\hline Technique & Application & Limitations \\
\hline DSA & $\begin{array}{l}\text { Gold-standard for PVD in elective and emergency } \\
\text { situations; both diagnostic and therapeutic }\end{array}$ & $\begin{array}{l}\text { Invasive, ionizing, contrast safety, sedation, luminal } \\
\text { imaging only }\end{array}$ \\
Duplex ultrasound & $\begin{array}{l}\text { Traditional first-line investigation for diagnostic } \\
\text { work-up of elective PVD patients; both diagnostic } \\
\text { and functional imaging }\end{array}$ & $\begin{array}{l}\text { Requires expertise, time-consuming, limited field of } \\
\text { view, calcification, stents }\end{array}$ \\
MRA & $\begin{array}{l}\text { Alternative first-line investigation for diagnostic } \\
\text { work-up of elective PVD patients }\end{array}$ & $\begin{array}{l}\text { Cost, magnetic safety, contrast safety, } \\
\text { claustrophobia, waiting list, calcification, stents }\end{array}$ \\
CTA & $\begin{array}{l}\text { Promising for diagnostic work-up of elective and } \\
\text { emergency PVD presentations }\end{array}$ & $\begin{array}{l}\text { Nontherapeutic in an emergency, ionizing, contrast, } \\
\text { data management (postprocessing) }\end{array}$ \\
\hline
\end{tabular}

Abbreviations: CTA, computed tomography angiography; DSA, digital subtraction angiography; MRA, magnetic resonance angiography; PVD, peripheral vascular disease.

problem, and could compensate for another modality's "blind spot". The imaging algorithm in Figure 5 provides a framework for the investigation of peripheral vascular disease.

Interestingly, a systematic review of the diagnostic accuracy of noninvasive imaging for detecting significant arterial stenosis (head-to-head with DSA as the gold-standard) has recently been published. After data was synthesized from 58 studies, contrast-enhanced MRA came out with the highest median sensitivity (95\%) and median specificity (97\%). CTA had a median sensitivity and specificity of $91 \%$, comparing favourably with DU (median sensitivity $88 \%$; median specificity $96 \%) .{ }^{45}$ However, in a response to this meta-analysis, it was claimed that DSA is consistently more accurate than all the other imaging modalities at assessing less than significant degrees of stenosis (ie, $<50 \%$ by diameter reduction), and that this additional ability was the reason why catheter angiography was the gold-standard for triaging patients into surgical and conservative management. ${ }^{46}$

\section{Conclusion}

Good patient outcomes depend on prompt diagnosis, intervention whilst emergencies are reversible, and appropriate long-term management. The choice between different imaging techniques also depends on local protocols, which will reflect the local population needs and the availability of local expertise and services. The strengths and weaknesses of the various imaging modalities also reveal how these modalities could complement each other. Table 2 summarizes the role of the various imaging modalities in the imaging of peripheral vascular disease. When investigating patients with one diagnostic modality, it is no longer possible to ignore how other modalities work.

\section{Disclosure}

The authors report no conflicts of interest in this work.

\section{References}

1. Hirsch AT, Haskal ZJ, Hertzer NR, et al; American Association for Vascular Surgery/Society for Vascular Surgery; Society for Cardiovascular Angiography and Interventions; Society for Vascular Medicine and Biology; Society of Interventional Radiology; ACC/ AHA Task Force on Practice Guidelines. ACC/AHA Guidelines for the Management of Patients with Peripheral Arterial Disease (lower extremity, renal, mesenteric, and abdominal aortic): a collaborative report from the American Associations for Vascular Surgery/Society for Vascular Surgery, Society for Cardiovascular Angiography and Interventions, Society for Vascular Medicine and Biology, Society of Interventional Radiology, and the ACC/AHA Task Force on Practice Guidelines (writing committee to develop guidelines for the management of patients with peripheral arterial disease) - summary of recommendations. J Vasc Interv Radiol. 2006;17(9):1383-1397.

2. Gjonnaess E, Morken B, Sandbaek G, et al. Gadolinium-enhanced magnetic resonance angiography, colour duplex and digital subtraction angiography of the lower limb arteries from the aorta to the tibioperoneal trunk in patients with intermittent claudication. Eur $J$ Vasc Endovasc Surg. 2006;31:53-58.

3. Griffin NMR, Wright IA, Buckenham TM. Comparison of duplex ultrasound with digital subtraction angiography in the assessment of infrainguinal autologous vein bypass grafts. ANZ J Surg. 2006;76:966-969.

4. Krnic A, Vucic N, Sucic Z. Duplex scanning compared with intraarterial angiography in diagnosing peripheral arterial disease: three analytical approaches. Vasa. 2006;35(2):86-91.

5. Wikstrom J, Holmberg A, Johansson L, et al. Gadolinium-enhanced MRA, DSA, and duplex of the iliac arteries compared with intra-arterial pressure gradient measurements. Eur J Vasc Endovasc Surg. 2000;19:516-523.

6. Tato F, Hoffmann U, Weber C, Reiser M, Rieger J. Comparison of angiography, duplex sonography, and intravascular ultrasound for the graduation of femoropopliteal stenoses before and after balloon angioplasty. Ultrasound Med Biol. 2006;32(12):1837-1843.

7. White C. Clinical practice: intermittent claudication. $N$ Engl J Med. 2007;356(12):1241-1250.

8. Moore WS, Hall AD. Unrecognized aortoiliac stenosis. A physiologic approach to the diagnosis. Arch Surg. 1971;103:633-638.

9. Hahn WY, Hecht EM, Friedman B, Babb JS, Jacobowitz GR, Lee VS Distal lower extremity imaging: prospective comparison of 2D TOF, 3D time-resolved ce-MRA, and 3D bolus chase ce-MRA. J Comput Assist Tomogr. 2007;31:29-36.

10. Thornton FJ, Du J, Suleiman SA, et al. High-resolution, time-resolved MRA provides superior definition of lower-extremity arterial segements compared to 2D TOF imaging. J Magn Reson Imaging. 2006;24:362-370.

11. Bezooijen R, van den Bosch HC, Tielbeek AV, et al. Peripheral arterial disease: sensitivity-encoded multiposition MR angiography compared with intraarterial angiography and conventional multiposition MR angiography. Radiology. 2004;231(1):263-271. 
12. Goyen M, Herborn CU, Kroger K, Ruehm SG, Debatin JF. Total-body 3D magnetic resonance angiography influences the management of patients with peripheral arterial occlusive disease. Eur Radiol. 2006;16(3):685-691.

13. Leiner T, Kessels AG, Nelemans PJ, et al. Peripheral arterial disease: comparison of color duplex US and contrast-enhanced MR angiography for diagnosis. Radiology. 2005;235:699-708.

14. Meissner OA, Verrel F, Tato F, et al. Magnetic resonance angiography in the follow-up of distal lower-extremity bypass surgery: comparison with duplex ultrasound and digital subtraction angiography. $J$ Vasc Interv Radiol. 2004;15(11):1269-1277.

15. de Vries M, Ouwendijk R, Flobbe K, et al. Peripheral arterial disease: clinical and cost comparisons between duplex US and contrastenhanced MR angiography - a multicenter randomised trial. Radiology. 2006;240:401-410.

16. Schulte AC, Bongartz G, Huegli R, et al. Intraarterial versus IV gadolinium injections for MR angiography: quantitative and qualitative assessment of the infrainguinal Arteries. AJR. 2005;185:735-740.

17. Huegli RW, Aschwanden M, Bongartz G, et al. Intraarterial MR angiography and DSA in patients with peripheral arterial occlusive disease: prospective comparison. Radiology. 2006;239(3):901-908.

18. Vogt FM, Ajaj W, Hunold P, et al. Venous compression at high-spatialresolution three-dimensional MR angiography of peripheral arteries. Radiology. 2004;233(3):913-920.

19. Tongdee R, Narra VR, McNeal G, et al. Hybrid peripheral 3D contrastenhanced MR angiography of calf and foot vasculature. AJR Am J Roentgenol. 2006;186(6):1746-1753.

20. Andreisek G, Pfammatter T, Goepfert K, et al. Peripheral arteries in diabetic patients: standard bolus-chase and time-resolved MR angiography. Radiology. 2007;242(2):610-620.

21. Ersoy H, Rybicki FJ. MR angiography of the lower extremities. AJR Am J Roentgenol. 2008;190(6):1675-1684.

22. Low G, Mizzi A, Ong K, Lau PF, McKinstery J. Technical inadequacies of peripheral contrast-enhanced MRA: incidence, causes and management strategies. Clinl Radiol. 2006;61:937-945.

23. Janka R, Wenkel E, Fellner C, Lang W, Bautz W, Uder M. Magnetic resonance angiography of the peripheral vessels in patients with peripheral arterial occlusive disease: when is an additional conventional angiography required? Cardiovasc Intervent Radiol. 2006;29:220-222.

24. Deutschmann HA, Schoellnast H, Portugaller HR, et al. Routine use of 3D contrast-enhanced moving-table MR angiography in patients with peripheral arterial occlusive disease: comparison with selective DSA. Cardiovasc Intervent Radiol. 2006;29:762-770.

25. Burrill J, Dabbagh Z, Gollub F, Hamady M. Multidetector computed tomographic angiography of the cardiovascular system. Postgrad Med J. 2007;83(985):698-704.

26. AbuRahma AF. Fate of endoleaks detected by CT angiography and missed by colour duplex ultrasound in endovascular grafts for abdominal aortic aneurysm. $J$ Endovasc Ther. 2006;13(4):490-495.

27. Armerding MD, Rubin GD, Beaulieu CF, et al. Aortic aneurysmal disease: assessment of stent-graft treatment-CT versus conventional angiography. Radiology. 2000;215:138-146.

28. Sun Z. Diagnostic accuracy of multislice CT angiography in peripheral arterial disease. J Vasc Interv Radiol. 2006;17:1915-1921.

29. Heijenbrok-Kal MH, Kock MC, Hunink MG. Lower extremity arterial disease: multidetector CT angiography meta-analysis. Radiology. 2007;245(2):433-439.

30. Met R, Bipat S, Legemate DA, Reekers JA, Koelemay MJW. Diagnostic performance of computed tomography angiography in peripheral arterial disease: a systematic review and meta-analysis. JAMA. 2009;301(4):415-424
31. Willmann JK, Baumert B, Schertler T, et al. Aortoiliac and lower extremity arteries assessed with 16-detector row CT angiography: prospective comparison with digital subtraction angiography. Radiology. 2005;236(3):1083-1093.

32. Laswed T, Rizzo E, Guntern D, et al. Assessment of occlusive arterial disease of abdominal aorta and lower extremities arteries: value of multidetector $\mathrm{CT}$ angiography using an adaptive acquisition method. Eur Radiol. 2008;18(2):263-272.

33. Schernthaner R, Stadler A, Lomoschitz F, et al. Multidetector CT angiography in the assessment of peripheral arterial occlusive disease: accuracy in detecting the severity, number, and length of stenoses. Eur Radiol. 2008;18(4):665-671.

34. Li XM, Xiao Y, Tian JM, Guang JZ, Tian JL, Gong J. The diagnostic value of 64-multislice $\mathrm{CT}$ in patients with peripheral arterial occlusive diseases: comparison with digital subtraction angiography. J Interv Radiol. 2007;16(6):371-374.

35. Li GC, Deng G, Qin YL, et al. The comparative study of 64-slices spiral CT angiography with DSA lower extremity arterial occlusive diseases. J Interv Radiol. 2008;17(5):336-339.

36. Bell KW, Heng RC, Atallah J, Chaitowitz I. Use of intra-arterial multi-detector row CT angiography for the evaluation of an ischaemic limb in a patient with renal impairment. Australas Radiol. 2006;50:377-380.

37. Fraioli F, Catalano C, Napoli A, et al. Low-dose multidetector-row CT angiography of the infra-renal aorta and lower extremity vessels: image quality and diagnostic accuracy in comparison with standard DSA. Eur Radiol. 2006;16(1):137-146.

38. Catalano C, Fraioli F, Laghi A, et al. Infrarenal aortic and lowerextremity arterial disease: diagnostic performance of multi-detector row CT angiography. Radiology. 2004;231(2):555-563.

39. Sarwar A, Rieber J, Mooyaart EA, et al. Calcified plaque: measurement of area at thin-section flat-panel CT and 64-section multidetector CT and comparison with histopathologic findings. Radiology. 2008; 249(1):301-306.

40. Martin ML, Tay KH, Flak B, et al. Multidetector CT angiography of the aortoiliac system and lower extremities: a prospective comparison with digital subtraction angiography. AJR Am J Roentgenol. 2003; 180(4):1085-1091.

41. Ouwendijk R, Kock MC, van Dijk LC, van Sambeek MR, Stijnen T, Hunink MG. Vessel wall calcifications at multi-detector row CT angiography in patients with peripheral arterial disease: effect on clinical utility and clinical predictors. Radiology. 2006;241:603-608.

42. Fishman EK, Horton KM, Johnson PT. Multidetector CT and threedimensional CT angiography for suspected vascular trauma of the extremities. Radiographics. 2008;28:653-667.

43. Yu H Zeng K, Bharkhada DK, et al. A segmentation-based method for metal artifact reduction. Acad Radiol. 2007;14(4):495-504.

44. Ouwendijk R, de Vries M, Stijnen T, et al. Multicenter randomized controlled trial of the costs and effects of noninvasive diagnostic imaging in patients with peripheral arterial disease: the DIPAD trial. AJR Am J Roentgenol. 2008;190(5):1349-1357.

45. Collins R, Cranny G, Burch J, et al. A systematic review of duplex ultrasound, magnetic resonance angiography and computed tomography angiography for the diagnosis and assessment of symptomatic, lower limb peripheral arterial disease. Health Technol Assess. 2007;11(20): iii-iv, xi-xiii, 1-184.

46. Kapoor S. Digital subtraction angiography: gold standard for detecting peripheral arterial disease. June 19, 2007. [Cited Aug 12, 2008]. BMJ Rapid Responses. Available from: http://www.bmj.com/cgi/content/ full/334/7606/1257. 